\title{
Optimization of semiconductor melts
}

\author{
Günter Bärwolff ${ }^{1}$ and Michael Hinze ${ }^{2}$ \\ 1 TU Berlin, Institute of Mathematics, D-10623 Berlin, Str. d. 17. Juni 135, Germany \\ 2 TU Dresden, Institute of Mathematics, D-01062 Dresden, Zellescher Weg 12-14, \\ Germany
}

\begin{abstract}
In the present paper we investigate optimal control of semiconductor melts in zone-melting and Czochralski growth configurations. The flow is governed by the Boussinesq approximation of the Navier-Stokes system. The control goal consists in tracking of a prescribed flow field. As control action boundary heating in terms of Dirichlet and Neumann-type boundary conditions is considered. Optimal control strategies are characterized in terms of the first-order optimality conditions. On the numerical level these optimality conditions are solved by a damped Picard iteration. We present numerical experiments in two and three spatial dimensions for the crystal $\left(B i_{0.25} S b_{0.75}\right)_{2} T e_{2}$, which is formed by a composition of bismuth point fifty antimony one point fifty tellurium two, as well as for $S i$ (Silicium).
\end{abstract}

\section{Introduction}

During the growth of crystals in axisymmetric zone melting devices the transition from the two-dimensional flow regime to an unsteady three-dimensional behavior of the velocity and temperature field is observed in experiments under certain conditions of the growth device. This behavior leads to so called striations which from the crystal quality point of view should be avoided during the growth process. To avoid such crystal defects it is important to figure out those parameters, which guarantee a stable steady two-dimensional melt flow during the growth process. There are several possibilities to determine these parameters. In the present work an optimization approach will be discussed.

From experiments and practical crystal production processes it is known that unsteady behavior of the melt and vortices near the fluid-solid-interphase decrease the crystal quality. From the optimization point of view it therefore makes sense to gain

(i) flows, which are nearly steady, and/or

(ii) flows, which only have small vorticity in a certain region of the melt zone.

In a mathematical setting the goal in (i) may be achieved by minimizing trackingtype functionals of the form

$$
J\left(\boldsymbol{u}, \theta_{c}\right)=\frac{1}{2} \int_{0}^{T} \int_{\Omega}|\boldsymbol{u}-\overline{\boldsymbol{u}}|^{2} d \Omega d t+\frac{\alpha}{2} \int_{0}^{T} \int_{\Gamma_{c}}\left(\theta_{c}^{2}+\theta_{c_{t}}^{2}\right) d \Gamma d t
$$

whereas goal (ii) may be related to minimal values of vorticity-type functionals of the form

$$
J\left(\boldsymbol{u}, \theta_{c}\right)=\frac{1}{2} \int_{0}^{T} \int_{\Omega}|\operatorname{curl} \boldsymbol{u}|^{2} d \Omega d t+\frac{\alpha}{2} \int_{0}^{T} \int_{\Gamma_{c}}\left(\theta_{c}^{2}+\theta_{c_{t}}^{2}\right) d \Gamma d t .
$$


Above, $\Omega \subset \mathbb{R}^{2,3}$ denotes the flow domain with the boundary $\Gamma$, and $[0, T]$ the time horizon. Further, $\boldsymbol{u}$ denotes the flow velocity vector field in the melt, and $\overline{\boldsymbol{u}}$ the desired state, which represents a physically favourable flow situation. The function $\theta_{c}$ denotes the temperature flux on the wall of the crucible and serves as control variable on the control boundary $\Gamma_{c}$. Both cost functionals contain two parts; the first part provides the mathematical formulation of the control gain, and the second part weighs the control cost with the parameter $\alpha>0$.

Above, and from now onwards derivatives w.r.t. time are denoted by the subscript $t$, i.e. $v_{t}:=\frac{\partial v}{\partial t}$.

Let us comment on related literature in optimal control of convective fluids. Optimal boundary temperature control of the Boussinesq approximation in two spatial dimensions is investigated by Abergel and Temam in [1]. Among other things the authors derive the first-order optimality conditions and present a convergence proof of the steepest descent method. An analytical framework for Robin-type boundary control of the velocity in three spatial dimensions is presented by Belmiloudi in [4]. The same author investigates regularity and optimal control problems of a perturbation of the Boussinesq system in [3]. Analytical contributions to the optimal control problem for the Boussinesq approximation in the stationary case are given by Lee and Imanuvilov [15,16], and for Dirichlet boundary control by Lee [14]. Model-predictive control of the Boussinesq approximation in two spatial dimensions, including several numerical experiments is presented by the second author together with Matthes in [10]. In [9] Gunzburger et al. optimize the crystal-melt complex in magnetic Czochralski growth for Si. As control parameters serve two real quantities, namely the Hartmann number and the slope of a linear boundary temperature profile on the crucible wall. Our approach differs from that taken by Gunzburger et al. in the choice of the design variables. We seek optimal boundary temperature control strategies, i.e. on the continuous level we have to deal with infinitely many design parameters, compared to only two in [9]. More details w.r.t. this concern are given at the end of Section 3. Finally we note that the present work extends the numerical investigations of zone melting presented by the first author, König and Seifert in $[2]$.

\section{Mathematical model}

The flow in the crystal melt is gouverned by the Boussinesq approximation of the Navier-Stokes system for the velocity $\boldsymbol{u}=(u, v, w)$, the pressure $p$ and the temperature $\theta$;

$$
\begin{aligned}
& \boldsymbol{u}_{t}+(\boldsymbol{u} \cdot \nabla) \boldsymbol{u}-\Delta \boldsymbol{u}+\nabla p-G r \theta \boldsymbol{g}=0 \text { on } \Omega_{T}, \\
& -\operatorname{div} \boldsymbol{u}=0 \text { on } \Omega_{T}, \\
& \left.\theta_{t}+\boldsymbol{u} \cdot \nabla \theta-\frac{1}{P r} \Delta \theta-f=0 \text { on } \Omega_{T} .\right\}
\end{aligned}
$$

Here $\boldsymbol{g}=(0,0,1)$, and $\Omega_{T}=\Omega \times(0, T)$ denotes the space-time cylinder with cylindrical melt zone of height $H$ and radius $R$. Furthermore, $G r$ denotes the Grashof number, and $\operatorname{Pr}$ the Prandtl number. Since in the present work we are 
mainly interested in control via boundary temperatures the absence of external forces is assumed.

System (3) is supplied with temperature boundary conditions of third kind on the crucible walls (which form the control boundary $\Gamma_{c}$ ), at the solid-liquid interface $\Gamma_{d}$ the melting temperature is prescribed, and Dirichlet boundary conditions at the remaining parts of the boundary. For the flow Dirichlet boundary conditions are prescribed on the whole boundary $\Gamma$. More precisely we set

$$
\left.\begin{array}{rlrl}
u=u_{d}, v=v_{d}, w & =w_{d} & & \text { on } \Gamma_{T}, \\
\lambda \frac{\partial \theta}{\partial \mathbf{n}}+\tilde{a}\left(\theta-\theta_{0}\right) & =\theta_{c} & & \text { on } \Gamma_{c T}, \\
\theta & =\theta_{d} & & \text { on } \Gamma_{d T}
\end{array}\right\}
$$

where $\Gamma_{T}:=\Gamma \times[0, T], \theta_{0}$ is some environmental temperature and $\lambda, \tilde{a}$ denote physical constants. From now onwards it is convenient to rewrite the boundary condition on $\Gamma_{c}$ in the form

$$
a \frac{\partial \theta}{\partial \mathbf{n}}+b \theta=\theta_{c} \quad \text { on } \quad \Gamma_{c T},
$$

with appropriate coefficients $a, b$ which may not vanish simultaneously. We note that it is possible to include via $u_{d}, v_{d}, w_{d}$ certain crystal and crucible rotations, as it is common in the case of Czochralski growth. In the case of zone melting techniques one would require $\boldsymbol{u}=\mathbf{0}$.

Finally let us discuss the initial values for (3). The initial velocity is chosen as the neutral position of the crystal melt, i.e.

$$
\boldsymbol{u}=\mathbf{0} .
$$

The initial temperature field is chosen as solution of

$$
-\frac{1}{P r} \Delta \theta=0 \text { in } \Omega, \theta=\theta_{0} \text { on } \Gamma_{c} \text {, and } \theta=\theta_{d} \text { on } \Gamma_{d} .
$$

The material properties and the dimensionless parameters depend on the specific application and have to be defined appropriately.

We assume that prescribing heat fluxes on the walls of the crucible is possible, so that boundary conditions of third kind can be utilized as control mechanism. The optimization goal then consists in finding an optimal boundary heating strategy by adjusting the heat fluxes. We note that once this optimal strategy is known, in a further step the methods developed in [12] may be applied to provide optimal heater locations by solving an appropriate inverse problem.

Let us note that the choice of $a \equiv 0, b \neq 0$ includes pure Dirichlet boundary control, and $a \neq 0, b \equiv 0$ pure Neumann boundary control. However, effects related to radiation are excluded.

Remark 1. A precise functional analytic setting of the Boussinesq approximation can be found in e.g. [1,3]. Existence and uniqueness of solutions to the Boussinesq approximation in the presence of boundary conditions of third kind for the temperature may be established following the lines of [18], compare also 
[10]. In the case of pure Dirichlet boundary conditions (i.e. $a \equiv 0$ ) the boundary values have to satisfy certain regularity requirements to guarantee the existence of weak solutions, see e.g. [1]. However, from the practical point of view it often is desirable to assume weaker regularity of controls, like e.g. only square integrability w.r.t. the spatial variables. For this type of controls (3)-(7) may be considered in the very-weak sense, i.e. formulated with the method of transposition [17]. The optimization problem (8) in this setting then is meaningful. We emphasize, that an analysis of optimal control problems for the problem classes considered in the present work in the very-weak setting is still missing. This to the best of our knowledge even is true for optimal boundary control problems of the Navier-Stokes system, compare [5, Section 2], where a detailed discussion of regularity aspects of boundary controls can be found.

\section{Optimization}

The optimization problem considered in the present work is given by

$$
\text { (P) } \quad\left\{\begin{array}{l}
\min J\left(\boldsymbol{u}, \theta_{c}\right) \\
\text { s.t. }(3)-(7)
\end{array}\right.
$$

To derive the first order necessary optimality conditions for this optimization problem we formally utilize the Lagrange technique. The related Lagrangian in the primitive setting is given by

$$
\begin{aligned}
& L\left(\boldsymbol{u}, p, \theta, \theta_{c}, \boldsymbol{\mu}, \xi, \kappa, \chi\right)=J\left(\boldsymbol{u}, \theta_{c}\right)+\left\langle\boldsymbol{\mu}, \boldsymbol{u}_{t}+(\boldsymbol{u} \cdot \nabla) \boldsymbol{u}-\Delta \boldsymbol{u}+\nabla p-G r \theta \boldsymbol{g}\right\rangle_{\Omega_{T}} \\
& -\langle\xi, \operatorname{div} \boldsymbol{u}\rangle_{\Omega_{T}}+\left\langle\kappa, \theta_{t}+\boldsymbol{u} \cdot \nabla \theta-\frac{1}{P r} \Delta \theta-f\right\rangle_{\Omega_{T}}+\left\langle\chi, a \frac{\partial \theta}{\partial \mathbf{n}}+b \theta-\theta_{c}\right\rangle_{\Gamma_{c T}},
\end{aligned}
$$

where $\langle\cdot, \cdot\rangle_{\Gamma_{c T}}$ and $\langle\cdot, \cdot\rangle_{\Omega_{T}}$ denote appropriate duality pairings, and $\boldsymbol{\mu}, \xi, \kappa$ and $\chi$ are Lagrange parameters. For example for $\boldsymbol{u}, p$ and $\theta$ sufficiently regular one has

$$
\begin{aligned}
& <\boldsymbol{\mu}, \boldsymbol{u}_{t}+(\boldsymbol{u} \cdot \nabla) \boldsymbol{u}-\Delta \boldsymbol{u}+\nabla p-G r \theta \boldsymbol{g}>_{\Omega_{T}}= \\
& \qquad \int_{\Omega_{T}}\left[\boldsymbol{u}_{t}+(\boldsymbol{u} \cdot \nabla) \boldsymbol{u}-\Delta \boldsymbol{u}+\nabla p-G r \theta \boldsymbol{g}\right] \cdot \boldsymbol{\mu} d \Omega d t
\end{aligned}
$$

The convergence analysis of the solution algorithms proposed in the subsequent sections will be given in a forthcoming paper. For a convergence proof of the steepest descent method applied to the solution of related optimal control problems we refer the reader to [1].

The necessary optimality conditions for $(\mathrm{P})$ are now given by

$$
\nabla L=0
$$


Assembling these conditions for the cost functions of (1) and (2) leads to the state equations (3)-(7), together with the so called adjoint system

$$
\begin{aligned}
& -\boldsymbol{\mu}_{t}-\Delta \boldsymbol{\mu}+(\nabla \boldsymbol{u})^{t} \boldsymbol{\mu}-(\boldsymbol{u} \cdot \nabla) \boldsymbol{\mu}+\nabla \xi=-\kappa \nabla \theta+\left\{\begin{array}{l}
-(\boldsymbol{u}-\overline{\boldsymbol{u}}) \\
\operatorname{curl} \operatorname{curl} \boldsymbol{u}
\end{array} \text { in } \Omega_{T},\right. \\
& -\operatorname{div} \boldsymbol{\mu}=0 \quad \text { in } \Omega_{T} \\
& \boldsymbol{\mu}=\mathbf{0} \quad \text { on } \Gamma_{T}, \\
& \boldsymbol{\mu}(T)=\mathbf{0} \quad \text { in } \Omega, \\
& -\kappa_{t}-\frac{1}{P r} \Delta \kappa-\boldsymbol{u} \cdot \nabla \kappa=G r \boldsymbol{g} \cdot \boldsymbol{\mu} \quad \text { in } \Omega_{T}, \\
& \kappa=0 \quad \text { on } \Gamma_{d T}, \\
& a \frac{\partial \kappa}{\partial \mathbf{n}}+b \kappa=0 \quad \text { on } \Gamma_{c T}, \\
& \kappa(T)=0 \quad \text { in } \Omega, \\
& \chi=\left\{\begin{aligned}
-\frac{1}{b P r} \frac{\partial \kappa}{\partial \mathbf{n}} & \text { if } b \neq 0 \\
\frac{1}{a P r} \kappa & \text { if } b=0
\end{aligned} \quad \text { on } \Gamma_{c T}\right.
\end{aligned}
$$

and the optimality conditions

$$
\begin{aligned}
\alpha\left(-\theta_{c_{t t}}+\theta_{c}\right) & =\chi & & \text { on } \Gamma_{c T} \\
\theta_{c}(0) & =\theta_{0} & & \text { on } \Gamma_{c}, \\
\theta_{c_{t}}(T) & =0 & & \text { on } \Gamma_{c} .
\end{aligned}
$$

Here $\theta_{0}$ denotes a temperature distribution on $\Gamma_{c}$ at the beginning of the melting process.

All together, the necessary optimality conditions for problem (P) form a boundary value problem for $\boldsymbol{u}, p, \theta, \boldsymbol{\mu}, \xi$, and $\theta_{c}$ w.r.t. space and time in the space-time domain $\Omega_{T}$, which inherits a very special structure.

From now onwards we assume that system (3) together with (4), (6) and (7) for given $\theta_{c}$ admits a unique solution (this is satisfied under appropriate assumptions at least in the two-dimensional case, see [7], [13]). Then the cost functionals in (1),(2) may be rewritten in the form

$$
\hat{J}\left(\theta_{c}\right)=J\left(\boldsymbol{u}\left(\theta_{c}\right), \theta_{c}\right),
$$

where the gradient of $\hat{J}$ is determined by the optimality condition (11). More precisely, there holds

$$
\hat{J}^{\prime}\left(\theta_{c}\right)=\alpha\left(-\theta_{c_{t t}}+\theta_{c}\right)-\chi
$$

To evaluate $\hat{J}^{\prime}\left(\theta_{c}\right)$ for given $\theta_{c}$ amounts to solving (3)-(7) for $\boldsymbol{u}, \theta$, and then (10) for $\boldsymbol{\mu}, \theta$ and $\chi$.

Let us note that second time derivatives in the expression $\hat{J}^{\prime}\left(\theta_{c}\right)$ can be avoided by introducing an auxiliary variable $\eta$, i.e.

$$
\theta_{c_{t}}=\eta \text { on } \Gamma_{c T} \text {. }
$$


This results in the optimality condition

$$
\begin{aligned}
& \theta_{c_{t}}=\frac{1}{\alpha} \zeta, \quad \theta_{c}(0)=\theta_{0} \quad \text { on } \Gamma_{c T} \\
& -\zeta_{t}=-\alpha \theta_{c}+\chi, \quad \zeta(T)=0 \quad \text { on } \Gamma_{c T},
\end{aligned}
$$

where $\zeta$ denotes the Lagrange multiplier for $(13)$. For $\hat{J}^{\prime}\left(\theta_{c}\right)$ we obtain

$$
\hat{J}^{\prime}\left(\theta_{c}\right)=\alpha\left(\theta_{c}-\eta_{t}\right)-\chi, \text { or with } \eta=\theta_{c_{t}}=\frac{1}{\alpha} \zeta, \quad \hat{J}^{\prime}\left(\theta_{c}\right)=\alpha \theta_{c}-\zeta_{t}-\chi .
$$

instead of (12). To anticipate discussion let us note that for our numerical experiments this and the approach based on (11) yield the same results.

Let us close this section with noting that the approach to boundary control presented in the present work is designed to compute temperature distributions at every single point of the control boundary, since it follows from (12) that the gradient of the cost functional w.r.t. $\theta_{c}$ can be expressed in terms of adjoint variables, so that the directional derivatives in all directions are available once the adjoint variables are determined. This is different to the approach presented in e.g. [9], were control functions are sought which only depend on a few numbers of parameters and directional derivatives w.r.t these parameters are computed using finite difference techniques. We note that the latter approach requires the solution of an auxiliary linear problem for every directional derivative, so that its computationally complexity is proportional to the number of parameters.

\section{The numerical approach}

We solve problem (8) by applying a damped Picard iteration to the KKT system (3)-(7), (10),(11). The pseudo-algorithm reads

i) choose $\theta_{c}^{0}$, set $\theta_{c}=\theta_{c}^{0}$,

ii) solve the forward problem for $[\boldsymbol{u}, \theta]\left(\theta_{c}\right)$

iii) solve the adjoint problem for $[\boldsymbol{\mu}, \kappa](\boldsymbol{u}, \theta)$

iv) update $\left.\theta_{c}^{+}:=\sigma_{r} \theta_{c}+\left(1-\sigma_{r}\right) \mathcal{H}^{-1}(\chi), \sigma_{r} \in\right] 0,1[$,

v) until convergence, set $\theta_{c}=\theta_{c}^{+}$, go to ii),

where $\mathcal{H}^{-1}(\chi)$ for given $\chi$ denotes the solution of (11). As stopping rule in $\mathrm{v}$ ) we use

$$
\frac{\left|\hat{J}\left(\theta_{c}^{+}\right)-\hat{J}\left(\theta_{c}\right)\right|}{\left|\hat{J}\left(\theta_{c}\right)\right|} \leq 10^{-5} .
$$

This condition is justified by the experiences from related problems in flow control that small changes in the control $\theta_{c}$ result in small changes of the objective functional.

Next let us describe the numerical solution methods used in ii)-iv). For this purpose we denote by $t_{i}:=i \tau, i=0, \ldots Z$ an equidistant time grid on $[0, T]$, where $\tau:=\frac{T}{Z}$ for some $Z \in \mathbb{N}$. Moreover, unknown quantities are supplied with superscripts. In ii) we apply a semi-implicit time discretization scheme. 
Convective terms are treated explicitly, conductive terms implicitly. We obtain for $n=0, \ldots, Z-1$

$$
\begin{aligned}
& \frac{\boldsymbol{u}^{n+1}}{\tau}-\Delta \boldsymbol{u}^{n+1}+\nabla p^{n+1}-G r \theta^{n+1} \boldsymbol{g}=\frac{\boldsymbol{u}}{\tau}-(\boldsymbol{u} \cdot \nabla) \boldsymbol{u}, \\
& -\operatorname{div} \boldsymbol{u}^{n+1}=0, \\
& \frac{\theta^{n+1}}{\tau}-\frac{1}{\operatorname{Pr}} \Delta \theta^{n+1}=\frac{\theta}{\tau}-(\boldsymbol{u} \cdot \nabla) \theta,
\end{aligned}
$$

supplied with the boundary conditions (4) at $t=t_{n+1}$. Here $\boldsymbol{u}$ and $\theta$ for $n=0$ are taken from (6) and (7), respectively. Of course, given $\boldsymbol{u}$ equation (16) can be solved for $\theta^{n+1}$. To solve (14),(15) we apply a pressure-correction scheme which is explained next. Taking the divergence in (14) leads to

$$
-\Delta p^{n+1}=\frac{1}{\tau} \operatorname{div} \hat{\boldsymbol{u}}
$$

where

$$
\hat{\boldsymbol{u}}=\boldsymbol{u}+\tau\left[(\boldsymbol{u} \cdot \nabla) \boldsymbol{u}+G r \theta^{n+1} \boldsymbol{g}\right] .
$$

For the pressure we take Neumann boundary conditions of the form $\frac{\partial p^{n+1}}{\partial \boldsymbol{n}}=0$ on $\Gamma \backslash \Gamma_{\text {ref }}$ and $\frac{\partial p^{n+1}}{\partial \boldsymbol{n}}=-M p^{n+1}$ on $\Gamma_{\text {ref }}$, with $M$ sufficiently large and $\left|\Gamma_{\text {ref }}\right|$ sufficiently small. On the numerical level this choice corresponds to fixing the pressure in one boundary grid point while prescribing homogeneous Neumann boundary conditions at the remaining boundary nodes.

Using these boundary conditions for $p^{n+1}$ equation (17) can be solved for $p^{n+1}$, which in turn determines the velocity field $\boldsymbol{u}^{n+1}$ in terms of

$$
\frac{1}{\tau} \boldsymbol{u}^{n+1}-\Delta \boldsymbol{u}^{n+1}=\frac{1}{\tau} \boldsymbol{u}-\nabla p^{n+1}
$$

together with boundary conditions for $\boldsymbol{u}^{n+1}$ from (4) for $t=t_{n+1}$. In summary step ii) amounts to solving one Poisson equation for $p^{n+1}$, three Helmholtz equations for $\boldsymbol{u}^{n+1}$, and one for $\theta^{n+1}$. Spatially these subproblems are discretized by the finite volume method on a staggered grid in cylindrical coordinates developed in [2]. The resulting linear systems are solved by ILU-preconditioned cg methods.

For the time discretization of the adjoint system in iii) we use the scheme

$$
\begin{aligned}
& \frac{\boldsymbol{\mu}^{n-1}-\boldsymbol{\mu}}{\tau}-\Delta \boldsymbol{\mu}^{n-1}+\left(\nabla \boldsymbol{u}^{n-1}\right)^{t} \boldsymbol{\mu}-\left(\boldsymbol{u}^{n-1} \cdot \nabla\right) \boldsymbol{\mu}+\nabla \xi^{n-1} \\
& =-\kappa \nabla \theta^{n-1}+\left\{\begin{array}{l}
-\left(\boldsymbol{u}^{n-1}-\overline{\boldsymbol{u}}\right) \\
\operatorname{curl} \operatorname{curl} \boldsymbol{u}^{n-1},
\end{array}\right. \\
& -\operatorname{div} \boldsymbol{\mu}^{n-1}=0, \\
& \frac{\kappa^{n-1}-\kappa}{\tau}-\boldsymbol{u}^{n-1} \cdot \nabla \kappa-\frac{1}{\operatorname{Pr}} \Delta \kappa^{n-1}=G r \boldsymbol{g} \cdot \boldsymbol{\mu}^{n-1},
\end{aligned}
$$

for $n=Z, \ldots, 1$, where we have $\boldsymbol{\mu}=\mu(T)=0$ and $\kappa=\kappa(T)=0$ for $n=Z$. The boundary conditions are taken from (10) for $t=t_{n-1}$. A motivation of 
this scheme together with a detailed discussion is given in the appendix. The quantities $\boldsymbol{\mu}^{n-1}, \xi^{n-1}$ are obtained from (20),(21) by the pressure-correction method explained above, and after that eq. (22) can be solved for $\kappa^{n-1}$. Spatially these subproblems are discretized again by the finite volume method of [2], and the resulting linear systems are also solved by ILU-preconditioned cg methods.

To provide $\mathcal{H}^{-1}(\chi)$ in iv) eq. (11) is solved for the control $\theta_{c}$ by a finite volume method in space and time, where the boundary conditions $\theta_{c}(\gamma, 0)=\theta_{c 0}$ and $\theta_{c_{t}}(\gamma, T)=0$ for $\gamma \in \Gamma_{c}$ are taken. We note that $\mathcal{H}:=-\partial_{t t}+i d$.

Let us note that for the computation of $\boldsymbol{\mu}^{n-1}, \xi^{n-1}, \kappa^{n-1}$ the flow $\boldsymbol{u}^{n-1}$ is required for $n=Z, \ldots, 1$. This means that we have to store these flow velocities in order to compute the adjoint solution $\boldsymbol{\mu}^{n-1}, \kappa^{n-1}$, and $\theta_{c}$. These amount of storage can be avoided by applying checkpointing techniques as proposed e.g. in $[8,11]$

Let us close this section with describing the numerical approach that we take to tackle (13)-(14). With the notations from above it reads

$$
\begin{aligned}
& \frac{\theta_{c}^{n+1}-\theta_{c}^{n}}{\tau}=\frac{1}{\alpha} \zeta^{n+1}, n=0, \ldots, Z-1, \theta^{0}=\theta_{0}, \\
& -\frac{\zeta^{n+1}-\zeta^{n}}{\tau}=-\alpha \theta_{c}^{n}+\chi^{n}, n=Z-1, \ldots, 0, \zeta^{Z}=0 .
\end{aligned}
$$

\section{Results of the numerical solution of the full problem}

\section{First test problem}

As a first test problem we consider a practical zone melting configuration with Dirichlet boundary control, i.e. we set $a \equiv 0, b:=1$. This configuration is investigated in [2].

We use the crystal $\left(B i_{0.25} S b_{0.75}\right)_{2} T e_{2}$, a composition of bismuth point fifty antimony one point fifty tellurium two, whose geometrical and material parameters are summarized in Table 1.

$\left(B i_{0.25} S b_{0.75}\right)_{2} T e_{2}$-crystals are used for small cooling devices. The Fig. 1 shows the physical domain of the melt zone. For the velocity we have homogeneous Dirichlet data on the whole boundary. For the temperature we have the bound-

\begin{tabular}{|l|l|l|}
\hline parameter & symbol & value \\
\hline radius of the ampulla & $R$ & $0.004 \mathrm{~m}$ \\
height of the melt & $H$ & $0.016 \mathrm{~m}$ \\
melting point temperature & $\theta_{s}$ & $613 \mathrm{~K}$ \\
thermal diffusivity & $a$ & $0.44000 \mathrm{e}-05 \frac{\mathrm{m}^{2}}{\mathrm{~s}_{2}}$ \\
kinematic viscosity & $\nu$ & $0.36310 \mathrm{e}-06 \frac{\mathrm{m}^{2}}{\mathrm{~s}}$ \\
thermal expansion coefficient & $\beta$ & $0.96000 \mathrm{e}-04 \mathrm{~K}^{-1}$ \\
\hline
\end{tabular}

Table 1. Parameters of $\left(B i_{0.25} S b_{0.75}\right)_{2} T e_{2}$-melt and of the melt geometry 

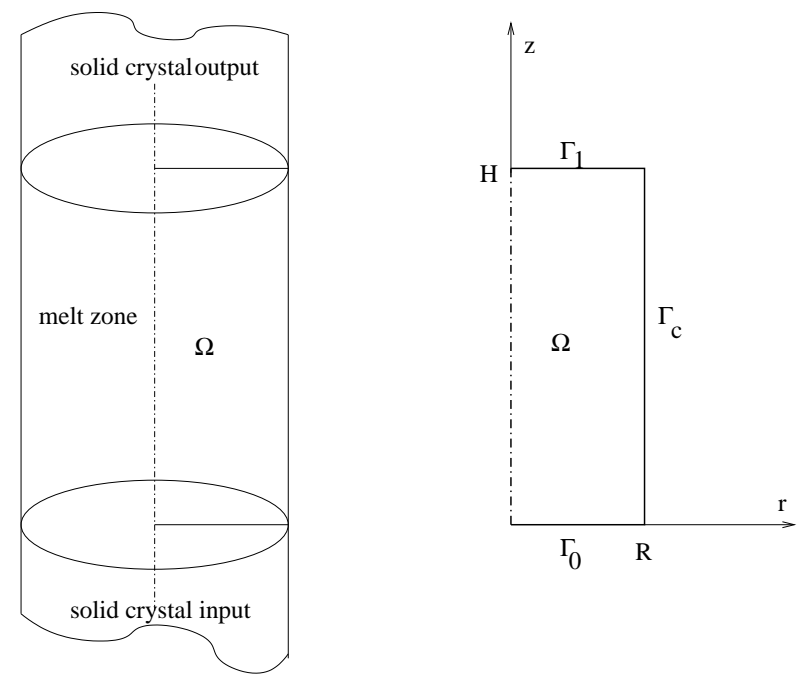

Fig. 1. Physical domain for the zone melting growth

ary conditions

$$
\begin{aligned}
& \theta=\theta_{c} \quad \text { for } r=R, 0 \leq z \leq H, \varphi \in(0,2 \pi), \quad\left(\text { control boundary } \Gamma_{c}\right) \\
& \theta=\theta_{s}, \quad \text { for } 0 \leq r \leq R, z=H, \\
& \theta=\theta_{s}, \quad \text { for } 0 \leq r \leq R, z=0 .
\end{aligned}
$$

For $t=0$ we start with a given temperature profile $\theta_{c}=\theta_{c 0}$ on $\Gamma_{c}$, and with $\theta_{s}=613 K$.For $\theta_{c 0}$ we have

$$
\theta_{c 0}(z)=\theta_{s}+4 \frac{z}{H}\left(1-\frac{z}{H}\right) \delta \theta
$$

with $\delta \theta=25 K$. The control goal is tracking of a velocity field $\overline{\boldsymbol{u}}$, which either is given by

i) a typical two-dimensional toroidal flow, or by

ii) a non moving melt, i.e. $\overline{\boldsymbol{u}}=\mathbf{0}$.

The case ii) is artificial but serves as a good test case since $\theta_{c}=\theta_{s}=$ const. implies $\boldsymbol{u}=\mathbf{0}$, and this velocity field together with $\theta=\theta_{s}$ is a solution of the Boussinesq approximation. Artificial in this context means that $\theta=\theta_{s}$ on $\Omega$ is not a realistic assumption for a crystal melt and the input mixed crystal will never change to a single homogeneous output crystal. We consider the time interval $[0, T]=[0,8$ seconds $]$ and $Z=60$ time steps of duration 0.1222 seconds each. For the spatial discretization we use a grid containing $20 \times 30$ finite volumes. As regularization and damping parameters we use $\alpha=0.25$ and $\sigma_{r}=0.1$. The Fig. 2 shows the results for case i), and Fig. 3 presents those for case ii). In both figures the left picture shows the development of the control temperature, 


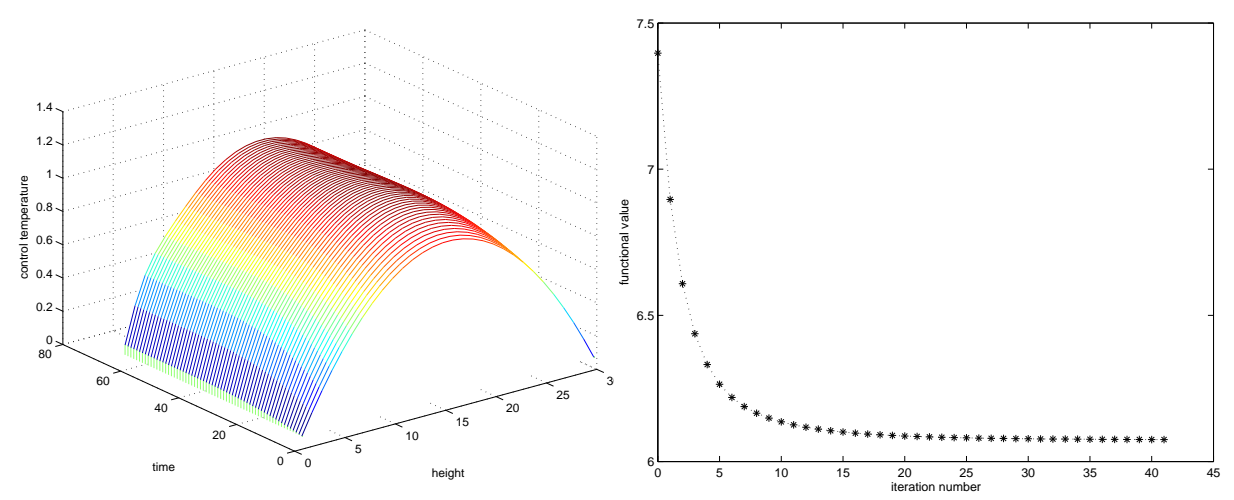

Fig. 2. Result of the zone melting process i)

whereas the right picture depicts the development of the functional value in relation to the iteration number of the Picard iteration. As can be seen the most significant reduction of the functional value already is achieved after the first few iterations. We note that the temperatures presented are dimensionless through the setting $\bar{\theta}=\frac{\theta-\theta_{s}}{\delta \theta}$. For the case i) the optimal control (see Fig. 2) is only slightly dependent of the time. We see only a slight increasing of the temperatures in the profiles $\theta_{c}(z)$ in the time direction. This behaviour can be explained by the fact that a forward simulation with constant in time temperature $\theta_{c}(z, t)=$ $\theta_{c 0}(z)$, with $\theta_{c 0}$ from (23) yields a velocity field $\boldsymbol{u}$ for which $\boldsymbol{u}(x, T)$ is very similar to $\overline{\boldsymbol{u}}(x)$.
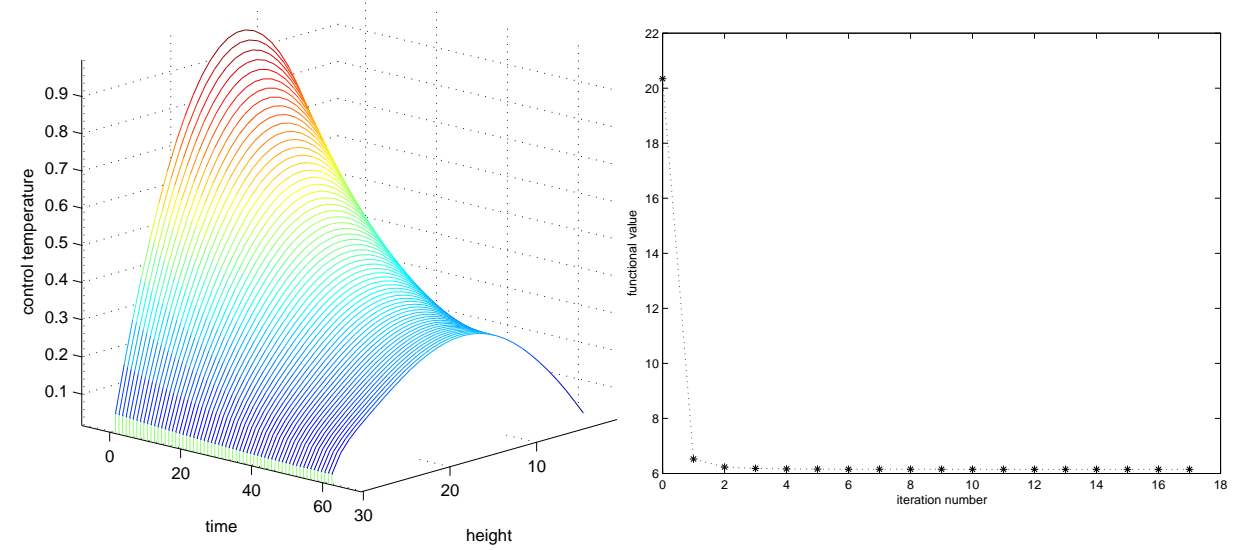

Fig. 3. Result of the zone melting process ii) 

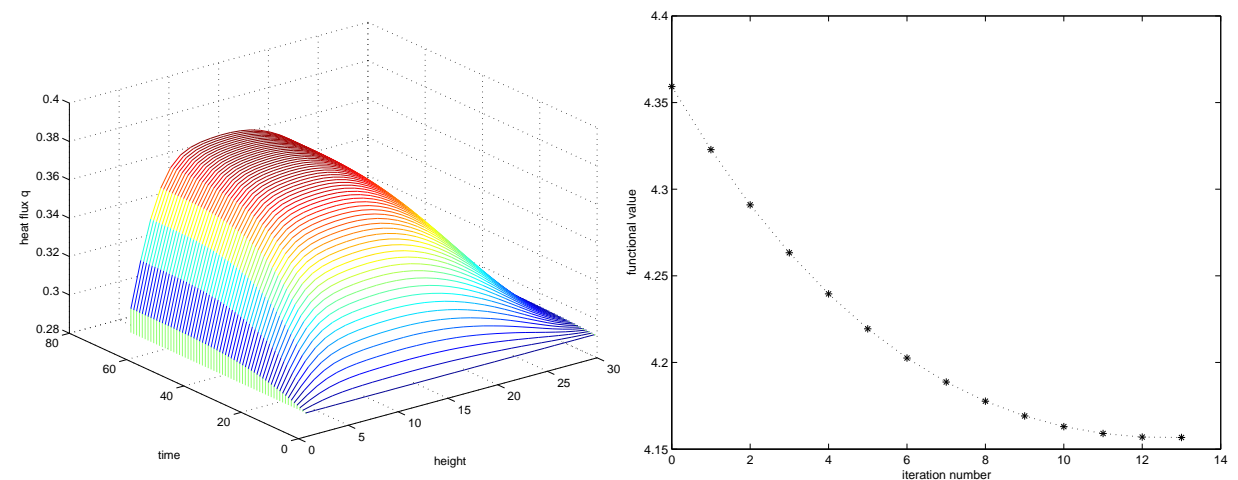

Fig. 4. Result of the optimization of the heating parameter $\overline{\theta_{c}}$ i)

Next we consider thermal boundary conditions of second kind, i.e. $b \equiv 0$, so that

$$
a \frac{\partial \theta}{\partial \mathbf{n}}=\theta_{c} \quad \text { for } r=R, 0 \leq z \leq H, \varphi \in(0,2 \pi),\left(\text { control boundary } \Gamma_{c}\right),
$$

and recall that for the adjoint temperature $\kappa$ we have on $\Gamma_{c}$ the boundary condition

$$
\frac{\partial \kappa}{\partial \mathbf{n}}=0 \text { for } r=R, 0 \leq z \leq H, \varphi \in(0,2 \pi) .
$$

We start with $a=\lambda=8,5 \frac{W}{m K}, \theta_{c}=\theta_{c_{0}}=13000 \frac{W}{m^{2}}$ and take the same geometrical and material parameters of the mixed crystal $\left(B i_{0.25} S b_{0.75}\right)_{2} T e_{2}$ as above.

As regularization parameter in the associated optimization problem we again take $\alpha=0.25$. For $\sigma_{r}$ we here apply a simple damping strategy which requires decrease of the functional value of at least $1 \%$ in every iteration of the solution process. Starting with $\sigma_{r}=0.05$ the damping parameter in case of failure of this test is reduced by the constant increment 0.01 . We note that for the present numerical experiment we have $\sigma_{r}=0.01$ in the final iteration. From our numerical tests we conclude that, compared to control by Dirichlet boundary conditions the Picard iteration in the case of Neumann boundary control is very sensitive with respect to damping.

The Fig. 4 shows the optimal $\overline{\theta_{c}}$ over the ampulla height and time (on $\Gamma_{c T}$ ) and the development of the functional values vs. the iteration counter, where the dimensionless control temperature $\overline{\theta_{c}}$ now is defined as

$$
\overline{\theta_{c}}=\frac{H \theta_{c}}{\delta \theta \lambda}
$$

If we set $\overline{\boldsymbol{u}}=\mathbf{0}$ we obtain the optimization results shown in Fig. 5. The heat flux tends to zero in time. This is what one expects in the present situation, since zero heat flux corresponds to constant temperature in the melt, and this 

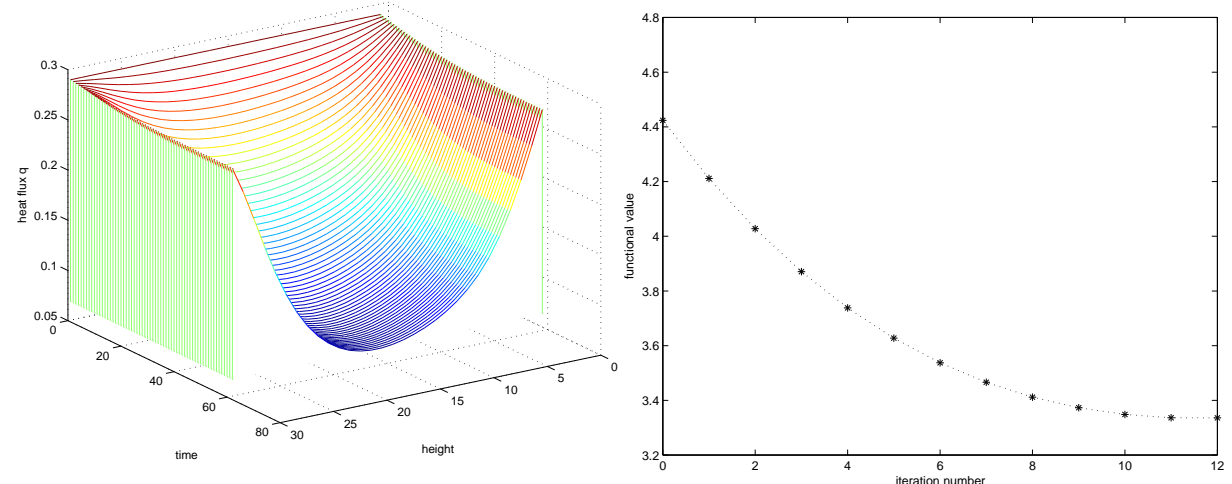

Fig. 5. Result of the optimization of the heating parameter $\overline{\theta_{c}}$ ii)

in turn to a non moving melt.

\section{Second test problem}

As a second test problem we consider a (idealized) Czochralski crystal growth process. The Fig. 6 shows the geometrical configuration of the crucible, which is taken from the benchmark in [19]. The above discussed model and the opti-
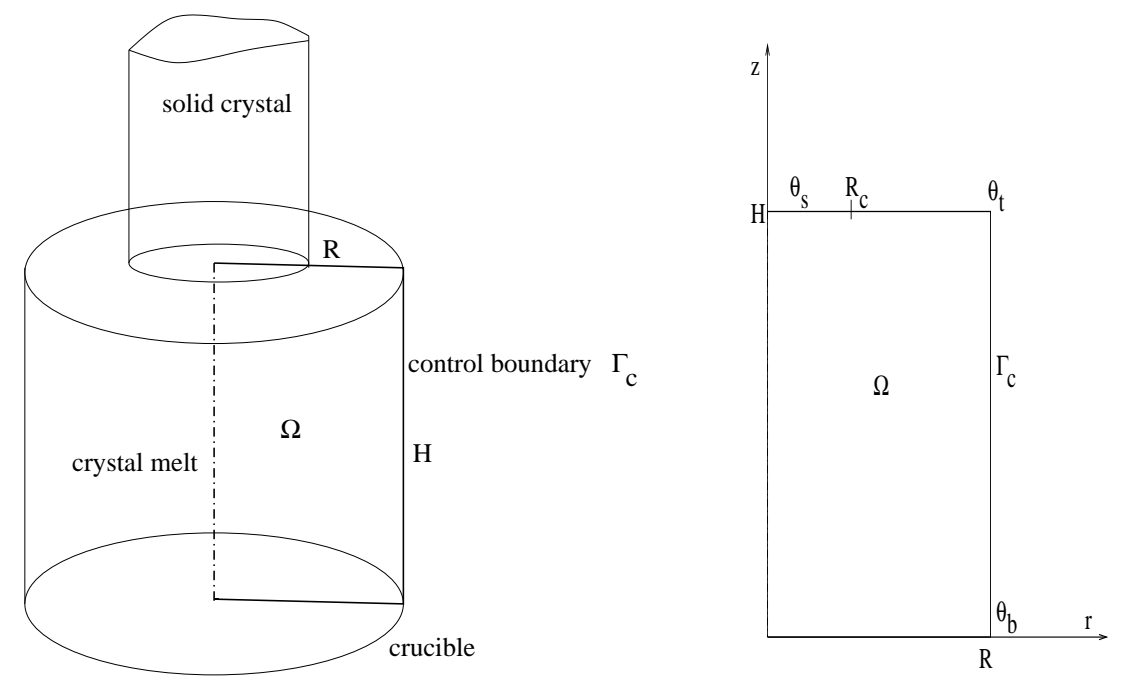

Fig. 6. Physical domain for Czochralski growth

mization system is formulated and implemented in three dimensions. However, because of the huge computational amount of work in three spatial dimensions 
we first test the optimization procedure for the two-dimensional case $u=0$ (azimuthal component of the velocity) and $\frac{\partial Q}{\partial \varphi}=0$ for all transport quantities $Q$ (u, $p, \theta$, etc.). Thus, we consider a two-dimensional spatial domain (see Fig. 6). $R_{c}$ denotes the radius of the solid crystal, $R$ the crucible radius and $H$ is the height of the crystal melt. $\theta_{s}$ denotes the melting point temperature of the crystal material, $\theta_{b}$ and $\theta_{t}$ are temperatures with $\theta_{b}>\theta_{t}>\theta_{s}$. The geometrical and material parameters are taken as in the Silicium Czochralski growth process described [6] and are summarized in the Table 2.

The associated Grashof number is given by $1.5 e+09$ and leads to a strong CFL restriction for time stepping in our time discretization scheme. Time steps $\tau$ should not be taken larger than $10^{-5}$. However, from the practical point of view this requirement is not as restrictive as it seems to be, since one dimensionless time step $\tau=10^{-5}$ corresponds to 0.80645 seconds real time.

Again we begin with investigating Dirichlet boundary control, i.e. we set $a \equiv 0$ and set $b:=1$. For the thermal boundary conditions of our Czochralski process we then have

$$
\begin{aligned}
& \theta=\theta_{c} \quad \text { for } r=R, 0 \leq z \leq H, \varphi \in(0,2 \pi),\left(\text { control boundary } \Gamma_{c}\right) \\
& \theta=\theta_{s}, \quad \text { for } 0 \leq r \leq R_{c}, z=H, \\
& \theta=\theta_{s}+\frac{r-R_{c}}{R-R_{c}}\left(\theta_{t}-\theta_{s}\right), \quad \text { for } R_{c} \leq r \leq R, z=H, \\
& \theta=\theta_{t}, \quad \text { for } 0 \leq r \leq R, z=0 .
\end{aligned}
$$

For $t=0$ we start with a given temperature profile $\theta_{c}=\theta_{c 0}$ on $\Gamma_{c}$, and with $\theta_{t}=1690 \mathrm{~K}, \theta_{b}=1708 \mathrm{~K}$ for $\theta_{c 0}$ we set

$$
\theta_{c 0}(z)=\theta_{b}+\frac{z}{H}\left(\theta_{t}-\theta_{b}\right) .
$$

As a desired velocity field we use $\overline{\boldsymbol{u}}=\mathbf{0}$. The spatial grid has the dimension $20 \times 45$. The Fig. 7, left shows the temperature distribution $\theta_{c}$ on $\Gamma_{c T}$ for a

\begin{tabular}{|c|c|c|}
\hline parameter & symbol & value \\
\hline crucible radius & $R$ & $0.15 \mathrm{~m}$ \\
\hline crystal radius & $R_{c}$ & $0.075 \mathrm{~m}$ \\
\hline height of the melt & $H$ & $0.4 \mathrm{~m}$ \\
\hline melting point temperature & $\theta_{s}$ & $1683 K$ \\
\hline thermal diffusivity & $a$ & $0.264 \mathrm{e}-04 \frac{m^{2}}{s}$ \\
\hline kinematic viscosity & $\nu$ & $0.279 \mathrm{e}-06 \frac{m^{2}}{s}$ \\
\hline thermal expansion coefficient & $\beta$ & $1.41^{*} 10^{-4} K^{-1}$ \\
\hline
\end{tabular}
time horizon containing 90 time steps ( $=68,4$ seconds). In this computations $\alpha=0.25$ is taken as regularization parameter, and the relaxation parameter is chosen $\sigma_{r}=0.75$. Fig. 7 , right compares the optimal control temperature profiles at time $t=0$ to those at $t=T$ for different regularization parameters $\alpha$. One

Table 2. Parameters of Silicium and of the melt geometry 

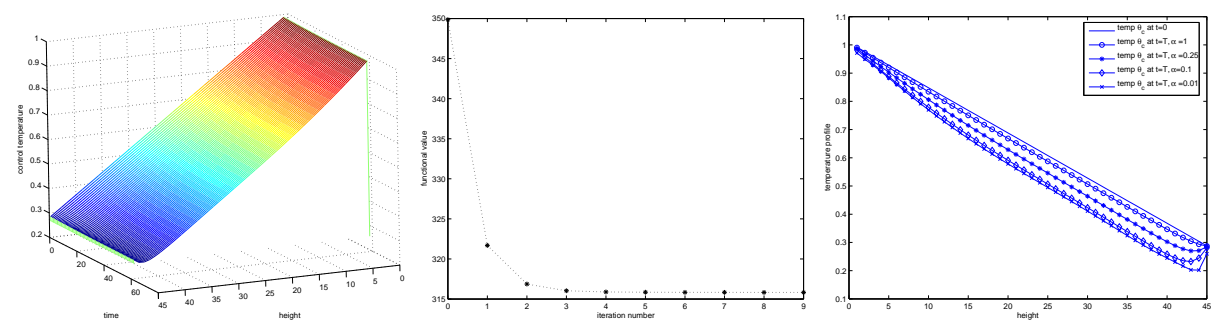

Fig. 7. Result of the optimization of the Czochralski process, second test problem

observes that the profiles at time $t=T$ become slightly nonlinear compared to the linear profile at $t=0$. The temperatures $\bar{\theta}$ in the Fig. 7 are dimensionless i.e.

$$
\bar{\theta}=\frac{\theta-\theta_{b}}{\theta_{t}-\theta_{b}} .
$$

Finally, we briefly report on the influence of the regularization parameter $\alpha$ on the control process. First we observe, that a reduction of $\alpha$ correlates to a decrease of the damping parameter $\sigma_{r}$ in the Picard iteration. In the present example we use $\sigma_{r}=0.9$ for $\alpha=1.0, \sigma_{r}=0.75$ for $\alpha=0.25, \sigma_{r}=0.4$ for $\alpha=0.1$, and $\sigma_{r}=0.1$ for $\alpha=0.01$.

However, it turns out that the quality of the controls does not change significantly with decreasing $\alpha$, at least in the investigated parameter range $\alpha \in$ $(0.01,1)$, compare Fig. 7 , right, where the final control temperature profiles at $t=T$ are presented. With the same figure we can report an increase of the gradient

$$
\left.\frac{\partial \theta_{c}}{\partial z}\right|_{\{z=H, t=T\}}
$$

at height $z=H$ and time $t=T$ with decreasing $\alpha$, while for $z \in(0,35)$ the temperature profiles remain nearly linear. These results to a certain extent justify the Ansatz of boundary temperature control for Czochralski growth presented in [9], where the slope of a linear boundary temperature profile serves as optimization variable. However, the Fig. 7, middle, where the development of the functional values during the optimization process for $\alpha=0.25$ and $\sigma_{r}=0.4$ is reported, shows in the present case $(\overline{\boldsymbol{u}}=\mathbf{0})$ a considerable decrease of the functional value.

For toroidal desired velocity fields we observe that the decrease of the functional value during the optimization process is very small or even negligible. This also is in accordance with the numerical findings of [9] for boundary temperature control.

\section{Third test problem}

As a three-dimensional test problem we consider the zone melting configuration of Fig. 1. The aim of the optimization is to track the velocity field $\overline{\boldsymbol{u}}=\mathbf{0}$. For the spatial discretization of the domain $[0,2 \pi] \times[0, R] \times[0, H]$ we use a $20 \times 20 \times 30$ 

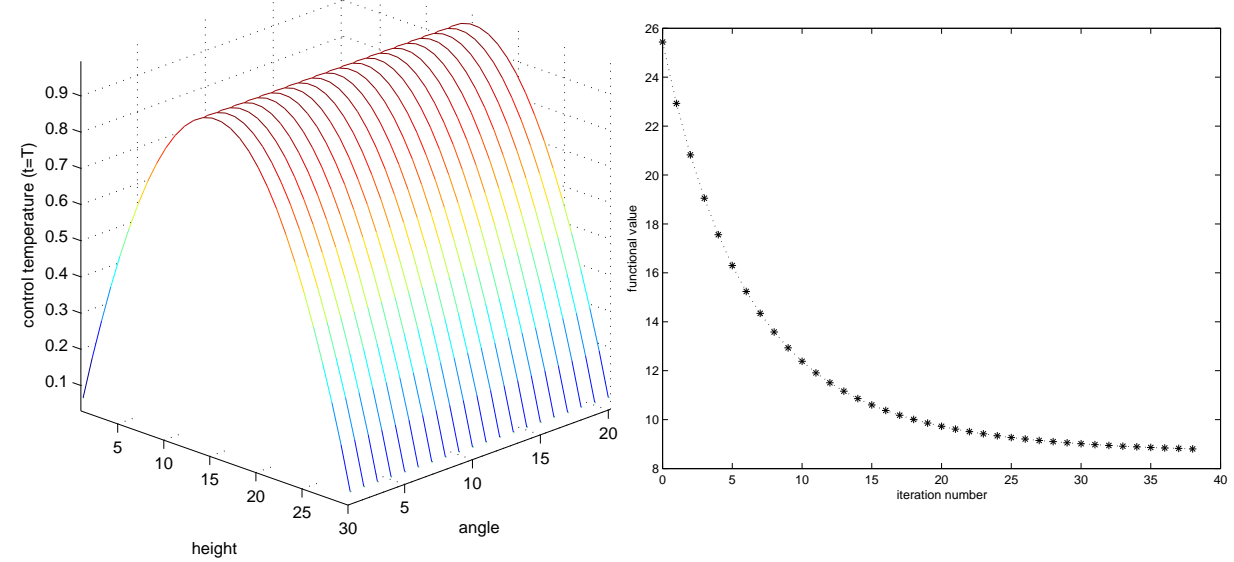

Fig. 8. Result of the optimization of Zone melting, third test problem

grid which is chosen equidistant in every coordinate direction. We consider the time interval $[0, T]=[0,4$ seconds $]$ with $Z=60$ time steps of duration 0.0661 seconds each. The parameters $\alpha=0.5$ and $\sigma_{r}=0.1$ are used for the threedimensional test problem. Fig. 8 shows the control temperature $\theta_{c}(\varphi, z, t)$ at the time $t=T$ on $\Gamma_{c}$ together with the development of the functional values during the optimization iterations. Note, that the temperature profile in Fig. 8 (left) shows $\theta_{c}(\varphi, z, T)$ for varying $\varphi$ and $z$. As one can see the profile is nearly constant in circumferential direction. Of course, this behavior is expected since $\overline{\boldsymbol{u}} \equiv \mathbf{0}$. Furthermore, in view of the first test problem, case ii), in the present test problem one now would expect a behaviour of $\theta_{c}(0, z, t)$ similar to that shown in Fig. 3. This in fact is the case, as Fig. 9 shows, where the control temperature $\theta_{c}(\varphi, z, t)$ on the line $\varphi=0=2 \pi$ is depicted.

\section{Conclusion}

Optimal boundary heating control strategies for fully time-dependent thermally coupled flow problems in spatially 3-dimensional cylindrical domains are developed. Optimal heating strategies are obtained as solutions of certain minimization problems and are computed from the related Karush-Kuhn-Tucker system by applying a damped Picard iteration.

Numerical results are presented for zone melting and Czochralski growth configurations in realistic 3 -dimensional cylindrical domains. While boundary heating control for zone melting configurations seems to offer a practically relevant control mechanism the numerical results for Czochralski growth indicate, that boundary heating for this configuration seems to have only limited impact on the flow behaviour in the melt. As a result for Czochralski growth other control mechanisms should be considered, like control by magnetic fields and/or crucible/crystal rotation. 


\section{Acknowledgments}

The first author acknowledges support of the Mathematisches Forschungszentrum Matheon granted by the Deutsche Forschungsgemeinschaft. The second author acknowledges support of the Sonderforschungsbereich 609 Elektromagnetische Strömungsbeeinflussung in Metallurgie, Kristallzüchtung und Elektrochemie granted by the Deutsche Forschungsgemeinschaft.

Finally, we thank the anonymous referees for many valuable comments which helped to improve the presentation of the material.

\section{References}

1. F. Abergel and R. Temam. On some Control Problems in Fluid Mechanics. Theoret. Comput. Fluid Dynamics, 1:303-325, 1990.

2. G. Bärwolff, G. König and G. Seifert. Thermal buoyancy convection in vertical zone melting configurations. ZAMM 77, 1997).

3. A. Belmiloudi. Regularity results and optimal control problems for the perturbation of the Boussinesq equations of the ocean. Numer. Funct. Anal., 21:623-651, 2000.

4. A. Belmiloudi. Robin-type boundary control problems for the nonlinear boussinesq type equations. J. Math. Anal. Appl., 273:428-456, 2002.

5. M. Berggren. Approximations of very weak solutions to boundary value problems. SIAM J. Numer. Anal., 42:860-877, 2004.

6. K. Böttcher. Collected solid crystal and crystal melt material parameters. Berlin, 2003 (available on the web page http://www.ikz-berlin.de/ ${ }^{k b}$ )

7. P. Constantin and C. Foias. Navier-Stokes Equations. The University of Chicago Press, 1988.

8. Griewank, A. and Walther, A. Revolve: An implementation of checkpointing for the reverse or adjoint mode of computational differentiation. ACM Trans. Math. Software, 26:19-45, 2000.

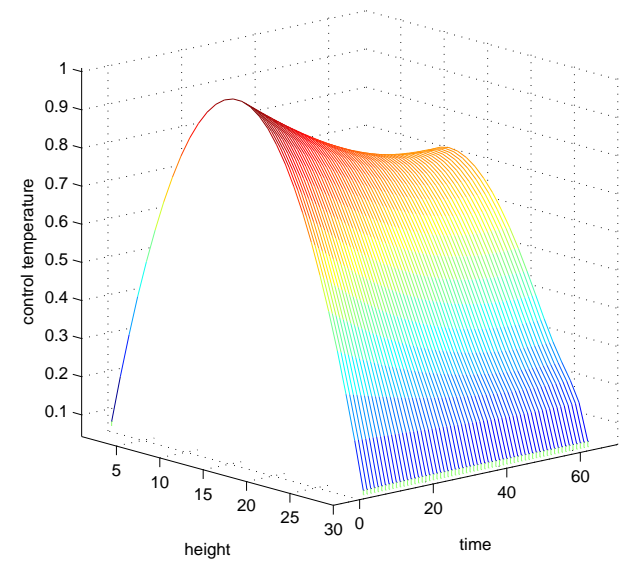

Fig. 9. control $\theta_{c}$ at $\varphi=0=2 \pi$ 
9. M. Gunzburger, E. Ozugurlu, J. Turner and H. Zhang. Controlling transport phenomena in the Czochralski crystal growth process. Journal of Crystal Growth, 234, 2002.

10. M. Hinze and U. Matthes. Optimal and model predictive control of the boussinesq approximation. Master's thesis, Institut für Numerische Mathematik, Technische Universität Dresden, 2004. Preprint MATH-NM-02-2004 (available on the web page

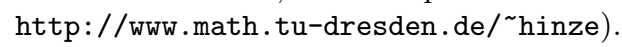

11. M. Hinze and J. Sternberg. A-Revolve: An adaptive memory- and run-timereduced procedure for calculating adjoints; with an application to the instationary Navier-Stokes system Preprint MATH-NM-01-2003 (available on the web page

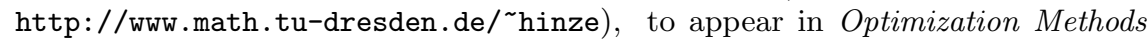
and Software.

12. M.R.H. Kurz and G. Müller. Control of thermal conditions during crystal growth by inverse modeling, Journal of Crystal Growth, 208, 2000.

13. O. Ladyzhenskaya. The Mathematical Theory of Viscous Incompressible Flows. Gordon \& Breach, 1969

14. H. Lee. Analysis and computational methods of Dirichlet boundary optimal control problems for $2 \mathrm{~d}$ boussinesq equations. Advances in Computational Mathematics, 19:255-275, 2003.

15. H. Lee and O.Y. Imanuvilov. Analysis of optimal control problems for the $2 \mathrm{~d}$ stationary boussinesq equations. J. Math. Anal. Appl., 242:191-211, 2000.

16. H. Lee and O.Y. Imanuvilov. Analysis of Neumann boundary optimal control problems for the stationary boussinesq equations including solid media. SIAM J. Control Optim., 39:457-477, 2000.

17. J.l. Lions and E. Magenes. Non-Homogeneous Boundary Value Problems and Applications. Springer-Verlag, Berlin, 1972.

18. R. Temam. Navier-Stokes Equations. North-Holland, 1979.

19. A.A. Wheeler. Four test problems for the numerical simulation of the CZ crystal growth. Journal of Crystal growth, 102, 1990. 


\section{Appendix}

\subsection{Construction of the adjoint time discretization}

The time discretization of the adjoint system presented in (20)-(22) is based on the transpose of semi-implicit time discretization of the derivative of the Boussinesq approximation. To become more precise let us write (3) in the form

$$
B(\boldsymbol{u}, p, \theta)=\left(\begin{array}{l}
\mathbf{0} \\
f
\end{array}\right) .
$$

Then the derivative of $B$ in direction $\tilde{\boldsymbol{u}}, \tilde{p}, \tilde{\theta}$ is given by

$$
B^{\prime}(\boldsymbol{u}, p, \theta)(\tilde{\boldsymbol{u}}, \tilde{p}, \tilde{\theta})=\left[\begin{array}{r}
\tilde{\boldsymbol{u}}_{t}-\Delta \tilde{\boldsymbol{u}}+(\boldsymbol{u} \cdot \nabla) \tilde{\boldsymbol{u}}+(\tilde{\boldsymbol{u}} \cdot \nabla) \boldsymbol{u}+\nabla \tilde{p}-G r \tilde{\theta} \boldsymbol{g} \\
-\operatorname{div} \tilde{\boldsymbol{u}} \\
\tilde{\theta}_{t}+\boldsymbol{u} \cdot \nabla \tilde{\theta}+\tilde{\boldsymbol{u}} \cdot \nabla \theta-\frac{1}{\operatorname{Pr}} \Delta \tilde{\theta}
\end{array}\right]
$$

The time discretization of (26) is adapted to that of the Boussinesq Approximation presented in (14)-(16). Again we denote by $t_{i}=i \tau, \tau=\frac{T}{Z}$ for some $Z \in \mathbb{N}$ an equidistant time grid. For $0 \leq n<Z$ we now set

$$
\begin{aligned}
& D_{\tau} B^{\prime}(\boldsymbol{u}, p, \theta)\left(\tilde{\boldsymbol{u}}^{n+1}, \tilde{p}^{n+1}, \tilde{\theta}^{n+1}\right)= \\
& \quad\left[\begin{array}{r}
\frac{\tilde{\boldsymbol{u}}^{n+1}-\tilde{\boldsymbol{u}}}{\tau}-\Delta \tilde{\boldsymbol{u}}^{n+1}+(\boldsymbol{u} \cdot \nabla) \tilde{\boldsymbol{u}}+(\tilde{\boldsymbol{u}} \cdot \nabla) \boldsymbol{u}+\nabla \tilde{p}^{n+1}-G r \tilde{\theta}^{n+1} \boldsymbol{g} \\
-\operatorname{div} \tilde{\boldsymbol{u}}^{n+1} \\
\frac{\tilde{\theta}^{n+1}-\tilde{\theta}}{\tau}+\boldsymbol{u} \cdot \nabla \tilde{\theta}+\tilde{\boldsymbol{u}} \cdot \nabla \theta-\frac{1}{\operatorname{Pr}} \Delta \tilde{\theta}^{n+1}
\end{array}\right],
\end{aligned}
$$

and note that all function values that appear without the superscript $n+1$ are taken at time instance $t=t_{n}$. Next we set

$$
\begin{gathered}
\mathcal{A}=\frac{1}{\tau} i d-\Delta, \quad \mathcal{B}_{n}=\frac{1}{\tau} i d-(\boldsymbol{u} \cdot \nabla)(\cdot)-((\cdot) \cdot \nabla) \boldsymbol{u}, \quad \mathcal{G}=\nabla, \quad \mathcal{F}=-G r \boldsymbol{g} \\
\mathcal{D}=-d i v, \quad \mathcal{K}=-(\cdot) \cdot \nabla \theta, \quad \mathcal{E}=\frac{1}{\tau} i d-\frac{1}{P r} \Delta, \mathcal{L}=\frac{1}{\tau} i d-\boldsymbol{u} \cdot \nabla
\end{gathered}
$$

and introduce the block operators

$$
A=\left(\begin{array}{ccc}
\mathcal{A} & \mathcal{G} & \mathcal{F} \\
\mathcal{D} & 0 & 0 \\
0 & 0 & \mathcal{E}
\end{array}\right) \quad \text { and } \quad B_{n}=\left(\begin{array}{ccc}
\mathcal{B}_{n} & 0 & 0 \\
0 & 0 & 0 \\
\mathcal{K} & 0 & \mathcal{L}
\end{array}\right)
$$

Further we set

$$
\mathbf{x}^{n+1}=\left(\begin{array}{c}
\tilde{\boldsymbol{u}}^{n+1} \\
\tilde{p}^{n+1} \\
\tilde{\theta}^{n+1}
\end{array}\right), \quad \mathbf{x}^{n}=\left(\begin{array}{c}
\tilde{\boldsymbol{u}} \\
\tilde{p} \\
\tilde{\theta}
\end{array}\right)
$$

and note that

$$
A \mathbf{x}^{0}=\left(\begin{array}{c}
\frac{\boldsymbol{u}_{0}}{\tau}-\Delta \boldsymbol{u}_{0}+\nabla p_{0}-G r \boldsymbol{g} \theta_{0} \\
0 \\
\frac{\theta_{0}}{\tau}-\Delta \theta_{0}
\end{array}\right)
$$


We are now prepared to rewrite the time discretization scheme (27) in the form

$$
\mathcal{M X}:=\left(\begin{array}{cccccc}
A & \mathbf{0} & & & & \\
-B_{0} & A & \mathbf{0} & & & \\
\mathbf{0} & -B_{1} & A & & & \\
\cdots & & & & & \\
& & & -B_{Z-2} & A & \mathbf{0} \\
& & & \mathbf{0} & -B_{Z-1} & A
\end{array}\right)\left(\begin{array}{c}
\mathbf{x}^{0} \\
\mathbf{x}^{1} \\
\mathbf{x}^{2} \\
\vdots \\
\mathbf{x}^{Z-1} \\
\mathbf{x}^{Z}
\end{array}\right)
$$

The transpose of $\mathcal{M}$ now defines the discretization scheme (20)-(22) of the adjoint system (10). More precisely, let

$$
\mathbf{y}^{n+1}=\left(\begin{array}{c}
\boldsymbol{\mu}^{n+1} \\
\xi^{n+1} \\
\kappa^{n+1}
\end{array}\right) \quad \text { and } \quad \mathbf{y}^{n}=\left(\begin{array}{c}
\boldsymbol{\mu} \\
\xi \\
\kappa
\end{array}\right)=\left(\begin{array}{c}
\boldsymbol{\mu}^{n} \\
\xi^{n} \\
\kappa^{n}
\end{array}\right),
$$

where $\boldsymbol{\mu}, \xi$ and $\kappa$ denote the adjoint variables, and set $\mathbf{Y}=\left(\mathbf{y}^{0}, \mathbf{y}^{1}, \ldots \mathbf{y}^{Z}\right)^{t}$. Then the time discretization scheme for the adjoint system is defined through

$$
(\mathcal{M X}, \mathbf{Y})=\left(\mathbf{X}, \mathcal{M}^{t} \mathbf{Y}\right)
$$

where

$$
\mathcal{M}^{t} \mathbf{Y}=\left(\begin{array}{cccccc}
A^{t}-B_{0}{ }^{t} & \mathbf{0} & & & \\
\mathbf{0} & A^{t} & -B_{1}{ }^{t} & \mathbf{0} & & \\
\cdots & & & & & \\
& & & A^{t}-B_{Z-2}{ }^{t} & \mathbf{0} \\
& & & \mathbf{0} & A^{t} & -B_{Z-1}{ }^{t} \\
& & & & \mathbf{0} & A^{t}
\end{array}\right)\left(\begin{array}{c}
\mathbf{y}^{0} \\
\mathbf{y}^{1} \\
\mathbf{y}^{2} \\
\vdots \\
\mathbf{y}^{Z-1} \\
\mathbf{y}^{Z}
\end{array}\right)
$$

Applying this time-discretization procedure to (10) we obtain for $n=Z, Z-$ $1, \ldots, 1$

$$
\begin{aligned}
& \frac{\boldsymbol{\mu}^{n-1}-\boldsymbol{\mu}}{\tau}+\left(\nabla \boldsymbol{u}^{n-1}\right)^{t} \boldsymbol{\mu}-\left(\boldsymbol{u}^{n-1} \cdot \nabla\right) \boldsymbol{\mu}-\Delta \boldsymbol{\mu}^{n-1}+\nabla \xi^{n-1} \\
& =-\kappa^{n-1} \nabla \theta+\left\{\begin{array}{l}
-\left(\boldsymbol{u}^{n-1}-\overline{\boldsymbol{u}}\right) \\
\operatorname{curl} \operatorname{curl} \boldsymbol{u}^{n+1},
\end{array}\right. \\
& \frac{\kappa^{n-1}-\kappa}{\tau}+\boldsymbol{u}^{n-1} \cdot \nabla \kappa-\frac{1}{\operatorname{Pr}} \Delta \kappa^{n-1}-G r \boldsymbol{g} \cdot \boldsymbol{\mu}^{n-1}=0
\end{aligned}
$$

which exactly represents (20)-(22).

\subsection{Cylindrical coordinates}

Since in our applications the Czochralski crucible and the zone melting ampulla have cylindrical geometry it is convenient to present the Boussinesq approxima- 
tion in cylindrical coordinates. W.r.t. these coordinates it has the form

$$
\begin{aligned}
& u_{t}+(r u u)_{r} / r+(u v)_{\varphi} / r+(w u)_{z}-v^{2} / r= \\
& -p_{r}+\left((r u)_{r} / r\right)_{r}+u_{\varphi \varphi} / r^{2}+u_{z z}-2 v_{\varphi} / r^{2}-u / r^{2}, \\
& v_{t}+(r u v)_{r} / r+(v v)_{\varphi} / r+(w v)_{z}+u v / r= \\
& -p_{\varphi} / r+\left((r v)_{r} / r\right)_{r}+v_{\varphi \varphi} / r^{2}+v_{z z}+2 u_{\varphi} / r^{2}-v / r^{2}, \\
& w_{t}+(r u w)_{r} / r+(v w)_{\varphi} / r+(w w)_{z}= \\
& -p_{z}+\left(r w_{r}\right)_{r} / r+w_{\varphi \varphi} / r^{2}+w_{z z}+G r \theta, \\
& (r u)_{r} / r+v_{\varphi} / r+w_{z}=0, \\
& \theta_{t}+(r u \theta)_{r} / r+(v \theta)_{\varphi} / r+(w \theta)_{z}=\frac{1}{\operatorname{Pr}}\left[\left(r \theta_{r}\right)_{r} / r+\theta_{\varphi \varphi} / r^{2}+\theta_{z z}\right] .
\end{aligned}
$$

To derive the adjoint system in cylindrical coordinates we set $\boldsymbol{\mu}=(\mu, \nu, \omega)$ where $\mu$ denotes the radial component, the azimuthal component is $\nu$, and the $z$-component is $\omega$. We now transfer the adjoint equations (10) to cylindrical coordinates and we get

$$
\begin{aligned}
& -\mu_{t}-\left((r \mu)_{r} / r\right)_{r}-\mu_{\varphi \varphi} / r^{2}-\mu_{z z}+2 \nu_{\varphi} / r^{2}+\mu / r^{2}+\mu(r u)_{r} / r+\nu v_{r} \\
& \quad+\omega w_{r}-(u \mu)_{r}-(v \mu)_{\varphi} / r-(w \mu)_{z}+v \nu / r+\xi_{r}=-(u-\bar{u})-\kappa \theta_{r} \\
& -\nu_{t}-\left((r \nu)_{r} / r\right)_{r}-\nu_{\varphi \varphi} / r^{2}-\nu_{z z}-2 \mu_{\varphi} / r^{2}+\nu / r^{2} \\
& \quad+\mu u_{\varphi} / r+\nu v_{\varphi} / r+\omega w_{\varphi} / r+(\nu u-2 \mu v) / r \\
& \quad-(r u \nu)_{r} / r+(v \nu)_{\varphi} / r-(w \nu)_{z}+\xi_{\varphi} / r=-(v-\bar{v})-\kappa \theta_{\varphi} / r \\
& -\omega_{t}-\left(r \omega_{r}\right)_{r} / r-\omega_{\varphi \varphi} / r^{2}-\omega_{z z}+\mu u_{z}+\nu v_{z}+\omega w_{z} \\
& \quad-(r u \omega)_{r} / r-(v \omega)_{\varphi} / r-(w \omega)_{z}+\xi_{z}=-(w-\bar{w})-\kappa \theta_{z}
\end{aligned}
$$

For the adjoint temperature $\kappa$ we get

$$
-\kappa_{t}-\frac{1}{P r}\left[\left(r \kappa_{r}\right)_{r} / r+\kappa_{\varphi \varphi} / r^{2}+\kappa_{z z}\right]-(r u \kappa)_{r} / r-(v \kappa)_{\varphi} / r-(w \kappa)_{z}=G r \omega,
$$

which is a convective heat conduction equation whose discretization can be performed as in [2], say. We note that one also would obtain (31)-(34) as the adjoint part of the optimality system, if in the definition of the Lagrangian in (9) cylindrical coordinates for the constitutive equations would be used, together with the volume element $d V=d x d y d z$ of the integrals replaced by $r d r d \varphi d z$.

Having in mind the spatial discretization of the Navier-Stokes system on a staggered grid the terms

$$
(\nabla \boldsymbol{u})^{t} \boldsymbol{\mu} \quad \text { and } \quad \kappa \nabla \theta
$$

occurring in (31)-(33) are not standard terms. Using a staggered grid finite volume method, $u$ and $\mu$ live at the same gridpoints, as do $v$ and $\nu, w$ and $\omega$, and $\theta$ and $\kappa$. Exemplarily we describe the discretization of the first component 
of both, $(\nabla \boldsymbol{u})^{t} \boldsymbol{\mu}$ and $\kappa \nabla \theta$. We obtain

$$
\begin{aligned}
& \left(\mu u_{r}+\nu v_{r}+\omega w_{r}\right)_{i+1 / 2 j k} \approx \\
& \mu_{i+1 / 2 j k}\left[\left(u_{i+3 / 2 j k}+u_{i+1 / 2 j k}\right)-\left(u_{i+1 / 2 j k}+u_{i-1 / 2 j k}\right)\right] /\left(2 \Delta x_{i+1 / 2}\right) \\
& +\nu_{i+1 / 2 j k}\left[\left(v_{i+1 j+1 / 2 k}+v_{i+1 j-1 / 2 k}\right)-\left(v_{i j+1 / 2 k}+v_{i j-1 / 2 k}\right)\right] /\left(2 \Delta x_{i+1 / 2}\right) \\
& +\omega_{i+1 / 2 j k}\left[\left(w_{i+1 j k+1 / 2}+w_{i+1 j k-1 / 2}\right)-\left(w_{i j k+1 / 2}+w_{i j k-1 / 2}\right)\right] /\left(2 \Delta x_{i+1 / 2}\right)
\end{aligned}
$$

where

$$
\begin{gathered}
\nu_{i+1 / 2 j k}=\left(\nu_{i j+1 / 2 k}+\nu_{i+1 j+1 / 2 k}+\nu_{i j-1 / 2 k}+\nu_{i+1 j-1 / 2 k}\right) / 4 \text { and } \\
\omega_{i+1 / 2 j k}=\left(\omega_{i+1 j k+1 / 2}+\omega_{i+1 j k-1 / 2}+\omega_{i j k+1 / 2}+\omega_{i j k-1 / 2}\right) / 4,
\end{gathered}
$$

and

$$
\kappa \theta_{r} \approx 0.5\left(\kappa_{i+1 j k}+\kappa_{i j k}\right)\left[\theta_{i+1 j k}-\theta_{i j k}\right] / \Delta x_{i+1 / 2} .
$$

